\title{
Stability of Model-Based Networked Control Systems With Time-Varying Transmission Times
}

\author{
Luis A. Montestruque, Member, IEEE, and Panos Antsaklis, Fellow, IEEE
}

\begin{abstract}
In model-based networked control systems (MB-NCSs), an explicit model of the plant is used to produce an estimate of the plant state behavior between transmission times. In this paper, the stability of MB-NCSs is studied when the controller/actuator is updated with the sensor information at nonconstant time intervals. Networked control systems with transmission times that are varying either within a time interval or are driven by a stochastic process with identically independently distributed and Markov-chain driven transmission times are studied. Sufficient conditions for Lyapunov stability are derived. For stochastically modeled transmission times almost sure stability and mean-square sufficient conditions for stability are introduced.
\end{abstract}

Index Terms-Model-based networked control systems (MB-NCSs), stochastic stability, time-varying transmission times.

\section{INTRODUCTION}

$\mathbf{T}$ HE study of control systems in which sensor data are fed back using a data network has recently received much attention. The use of a data network in the feedback path has several advantages such as reconfigurability, low installation cost, and easy maintenance; it is also well suited for large geographically distributed systems. There are, nevertheless, some shortcomings to the use of digital networks in control systems such as the fact that data networks operate in a discrete fashion delivering information only at specific instants in time. This means that the controller cannot have access to the plant output at all times. Moreover, increasing the availability of the output information to the controller means increasing the bandwidth needed by the control system. Even for discrete plants where the controller acts only at specific instants of time, if the sensor data is sent through the network at each sampling time and the sampling rate is low, the bandwidth required might still be large.

There is a tradeoff between the stability and performance of the control system and bandwidth usage. Good performance and large stability margins can be obtained by having accurate sensor measurements that are communicated to the controller in a timely manner, and so good performance and stability can be achieved at the expense of large network bandwidth usage.

Manuscript received May 19, 2003; revised March 21, 2004. Recommended by Guest Editors P. Antsaklis and J. Baillieul. This work was supported in part by the National Science Foundation under Grants NSF CCR01-13131 and ECS02-25265, and in part by the DARPA/IXO-NEST Program under Grant AF-F30602-01-2-0526.

The authors are with the Department of Electrical Engineering, University of Notre Dame, Notre Dame, IN 46556 USA (e-mail: 1montest@nd.edu; pantsakl@nd.edu).

Digital Object Identifier 10.1109/TAC.2004.834107
It is clear that the reduction of bandwidth necessitated by the communication network in a networked control system is a major concern. The available bandwidth restricts the number of bits per second (bit rate). Note that the bit rate is determined by two parameters: The transmission times or how often the measured signal is sampled and transmitted through the network; and the number of bits per measurement or how accurately the transmitted value is represented in digital form. Therefore, the networked control bandwidth reduction can perhaps be addressed by two methods: The first method is to minimize the transfer of information between the sensor and the controller/actuator; the second method is to compress or reduce the size of the data transferred at each transaction.

To answer this question it is perhaps useful to recall some of the characteristics of popular industrial network protocols. These are small transport time and large overhead (network control information included in the packet). This means that data compression by reducing the size of the data transmitted has negligible effects over the overall system performance in such type of networks. So, reducing the number of packets transmitted appears to bring more benefits than data compression. The reduction of the number of packets transmitted through the network can translate into larger minimal transfer times between the components. It is also to be noted that any delay in an information transaction is usually due to network access contention. Thus, a sensor with a fast sampling rate can send through the network the latest data available resulting in a negligible information transfer delay. However, there will still be contention in the network so that, even though the delay is small, the sensor data would not be available at all times to the controller/actuator. This brings us again to the idea of reducing the data transfer rate as much as possible. In this manner, more bandwidth will be available to allocate more resources without sacrificing stability and ultimately performance of the overall system.

The MB-NCS architecture of interest was introduced in [11]. The MB-NCS uses an explicit model of the plant to estimate the plant state between transmission times and generate the appropriate control signals; see Fig. 1. At each transmission time the plant model is updated with the measured plant state. This control architecture has as its main objective the reduction of the data packets transmitted over the network. In this way, the amount of bandwidth necessary for feedback control to maintain certain stability and performance criteria is minimized. Note that the fact that a model is included in the actuator side of the plant to be controlled is reasonable and practical.

It is intuitively clear that stability margins, controller robustness, and other performance indices will be improved when knowledge about the plant dynamics is used to predict plant behavior and appropriately modify the control input when the 


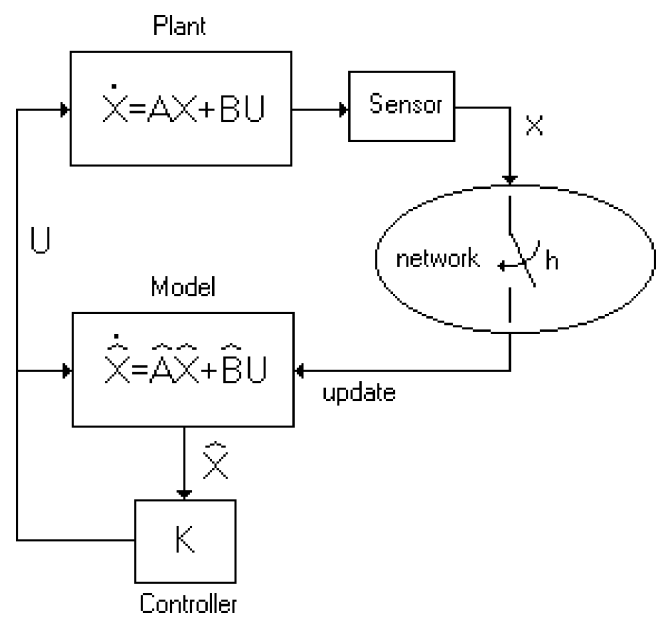

Fig. 1. State feedback model-based networked control system.

sensor information is not available and the plant is running open loop.

The use of an explicit model of the plant dynamics in the controller is not new. In the model predictive control [1] approach, for example, an explicit model of the plant to be controlled is used. The model is used to predict the future output behavior. This prediction capability allows solving optimal control problems online, where tracking error, namely the difference between the predicted output and the desired reference, is minimized over a time horizon, possibly subject to constraints on the manipulated inputs and outputs. Another control technique known as internal model control [15] uses a plant model to subtract the effect of the manipulated variables from the plant output. That is, assuming the plant is stable, the model is used to obtain a measure of the disturbances affecting the plant and of the inaccuracies of the model. This signal is then used to feed the so-called IMC controller. We use a plant model in MB-NCS to predict the output of the plant so that more accurate information can be fed to the controller when this information is not directly available via measurements.

In this paper, we study the state feedback MB-NCS shown in Fig. 1. We will assume that data for the whole state vector are sent by the sensor every $h(k)$ units of time and that the transmission time delay is negligible. The present paper represents a significant extension of previous results that involved constant update times. Specifically, in [14], MB-NCSs with state feedback, output feedback, network delays, or discrete plant are studied. In the output feedback MB-NCS, where a state observer estimates the value of the plant state vector and uses it to update the model, it is shown that the separation principle does not always hold. The case where network delays exist is also considered; it this case the delays and the update times are assumed to be constant. A "propagation unit" is introduced before the model update to estimate the actual value of the plant state vector. Finally extensions to the case of discrete plants are considered. The necessary and sufficient stability results obtained for these constant update times are similar: they require that the eigenvalues of a test matrix lie inside the unit circle. These test matrices also have similar structures.
In Fig. 1, the packets transmitted by the sensor contain the measured value of the plant state vector and are used to update the plant model on the actuator/controller node. These packets are transmitted at times $t_{k}$. We define the update times as the times between transmissions or model updates: $h(k)=t_{k+1}-$ $t_{k}$. In [11], [12], and [14], we made the assumption that the update times $h(k)$ are constant. This might not always be the case in real world applications. The transmission times of data packets from the plant output to the controller/actuator might not be periodic due to network contention or to the nondeterministic nature of the transmitter task execution scheduler. Soft realtime constraints provide a way to enforce the execution of tasks in the transmitter microprocessor. This allows the task of periodically transmitting the plant information to the controller/actuator to be executed at times $t_{k}$ that can vary according to certain predetermined stochastic process. Note that most work on networked control systems assumes deterministic communication rates [6], [7] or time-varying rates without considering the stochastic behavior of these rates [10], [19]. Little work has been done for the case where the update times have some stochastic properties.

Yook et al. [18] also approach the problem of reducing the bandwidth utilization by making use of a plant model; here the update of the model is event driven as opposed to time driven. The model is updated when any of the states differ from the computed value for more than a certain threshold. Some stability and performance conditions are derived as functions of the plant, threshold, and magnitude of the plant-model mismatch.

In [17], Ling and Lemmon study the performance of a networked control system. The performance index considered is the power spectral density of the plant output. The plant is assumed to be discrete and the network may drop measurement packets according to an independently identically distributed process with a specified drop out rate. The networked control system considered in [17] transmits measurement packets at every clock tic and no plant model is placed at the controller/actuator side.

In [16] Nair et al. consider a fully observed scalar Markov jump linear plant. The sensor encodes information about the plant state and mode and transmits it to the controller/actuator. The results obtained in [16] characterize the form of the encoder-decoder pair in order to achieve stability.

In this paper, we first obtain, in Section II, the response of the state feedback MB-NCS shown in Fig. 1. Then we study its stability properties assuming that the update times can take any values in an interval $\left[h_{\min }, h_{\max }\right]$. In this case, we will assume that we do not have any statistical knowledge about the update times. We analyze the stability properties of this system using Lyapunov techniques. This is the strongest type of stability presented and will provide a first cut on the characterization of the stability properties perhaps for comparison purposes. This type of stability is addressed in Section III.

Next, two types of stochastic stability are discussed, namely almost sure or probability-1 asymptotic stability in Section IV and mean-square or quadratic asymptotic stability in Section V. The first one is the one that mostly resembles deterministic stability [8]. Mean-square stability is attractive since it is related to optimal control problems such as the linear quadratic regulator (LQR). 
Two different types of time-varying transmission times are considered for each case in Sections IV and V. The first assumes that the transmission times are independent identically distributed with probability distribution function that may have support for infinite update times. The second type of stochastic update time assumes that the transmission times are driven by a finite Markov chain. Both models are common ways of representing the behavior of network transmission and scheduler execution times. Note that an early version of some of the results in this paper were presented in [13].

\section{State Feedback Model-Based Networked Control SYSTEM RESPONSE}

The dynamics of the system shown in Fig. 1 are given by

$$
\begin{aligned}
& {\left[\begin{array}{c}
\dot{x}(t) \\
\dot{e}(t)
\end{array}\right]=} {\left[\begin{array}{cc}
A+B K & -B K \\
\tilde{A}+\tilde{B} K & \hat{A}-\tilde{B} K
\end{array}\right]\left[\begin{array}{c}
x(t) \\
e(t)
\end{array}\right] } \\
& {\left[\begin{array}{c}
x\left(t_{k}\right) \\
e\left(t_{k}\right)
\end{array}\right]=} {\left[\begin{array}{c}
x\left(t_{k}^{-}\right) \\
0
\end{array}\right] \quad \forall t \in\left[t_{k}, t_{k+1}\right), } \\
& \text { with } t_{k+1}-t_{k}=h(k)
\end{aligned}
$$

where $e(t)=x(t)-\hat{x}(t)$ represents the error between the plant state and the plant model state, $A$ and $B$ are the matrices of the actual plant state-space representation, $\hat{A}$ and $\hat{B}$ are the matrices of plant model state-space representation, and $\tilde{A}=A-\hat{A}$ and $\tilde{B}=B-\hat{B}$ represent the modeling error matrices. Define $z(t)=\left[\begin{array}{ll}x(t)^{T} & e(t)^{T}\end{array}\right]^{T}$, and $\Lambda=\left[\begin{array}{cc}A+B K & -B K \\ \tilde{A}+\tilde{B} K & \hat{A}-\tilde{B} K\end{array}\right]$ so that (1) can be rewritten as $\dot{z}=\Lambda z$ for $t \in\left[t_{k}, t_{k+1}\right)$.

Proposition 1: The system described by (1) with initial conditions $z\left(t_{0}\right)=\left[\begin{array}{ll}x\left(t_{0}\right) & 0\end{array}\right]^{T}=z_{0}$ has the following response:

$$
\begin{gathered}
z(t)=e^{\Lambda\left(t-t_{k}\right)}\left(\prod_{j=1}^{k} M(j)\right) z_{0} \\
\text { for } t \in\left[t_{k}, t_{k+1}\right), t_{k+1}-t_{k}=h(k), \\
\text { where } M(j)=\left[\begin{array}{ll}
I & 0 \\
0 & 0
\end{array}\right] e^{\Lambda h(j)}\left[\begin{array}{ll}
I & 0 \\
0 & 0
\end{array}\right] .
\end{gathered}
$$

Proof: The proof is similar to the corresponding proof for constant $h$ in [11] and it is included here for completeness. On the interval $t \in\left[t_{k}, t_{k+1}\right)$, the system response is

$$
z(t)=\left[\begin{array}{c}
x(t) \\
e(t)
\end{array}\right]=e^{\Lambda\left(t-t_{k}\right)}\left[\begin{array}{c}
x\left(t_{k}\right) \\
0
\end{array}\right]=e^{\Lambda\left(t-t_{k}\right)} z\left(t_{k}\right)
$$

Now, note that at times $t_{k}, z\left(t_{k}\right)=\left[\begin{array}{c}x\left(t_{k}\right) \\ 0\end{array}\right]$, that is, the error $e(t)$ is reset to zero. We can represent this by

$$
z\left(t_{k}\right)=\left[\begin{array}{ll}
I & 0 \\
0 & 0
\end{array}\right] z\left(t_{k}^{-}\right)
$$

Using (3) to calculate $z\left(t_{k}^{-}\right)$, we obtain

$$
z\left(t_{k}\right)=\left[\begin{array}{ll}
I & 0 \\
0 & 0
\end{array}\right] e^{\Lambda h(k)} z\left(t_{k-1}\right)
$$

In view of (3), we have that if at time $t=t_{0}, z\left(t_{0}\right)=z_{0}=\left[\begin{array}{c}x_{0} \\ 0\end{array}\right]$ is the initial condition then

$$
\begin{aligned}
z(t) & =e^{\Lambda\left(t-t_{k}\right)} z\left(t_{k}\right) \\
& =e^{\Lambda\left(t-t_{k}\right)}\left[\begin{array}{ll}
I & 0 \\
0 & 0
\end{array}\right] e^{\Lambda h(k)} z\left(t_{k-1}\right) \\
& =e^{\Lambda\left(t-t_{k}\right)}\left[\begin{array}{ll}
I & 0 \\
0 & 0
\end{array}\right] e^{\Lambda h(k)}\left[\begin{array}{ll}
I & 0 \\
0 & 0
\end{array}\right] e^{\Lambda h(k-1)} z\left(t_{k-2}\right) \\
& =e^{\Lambda\left(t-t_{k}\right)}\left(\prod_{j=1}^{k}\left[\begin{array}{ll}
I & 0 \\
0 & 0
\end{array}\right] e^{\Lambda h(j)}\right) z_{0} .
\end{aligned}
$$

Now, we know that $\left[\begin{array}{ll}I & 0 \\ 0 & 0\end{array}\right] e^{\Lambda h(j)}$ is of the form $\left[\begin{array}{cc}S_{j} & P_{j} \\ 0 & 0\end{array}\right]$ and so $\left(\prod_{j=1}^{k}\left[\begin{array}{ll}I & 0 \\ 0 & 0\end{array}\right] e^{\Lambda h(j)}\right)$ has the form $\left[\begin{array}{cc}\left(\prod_{j=1}^{k} S_{j}\right) & \cdot \\ 0 & 0\end{array}\right]$. Additionally, we note the special form of the initial condition $z\left(t_{0}\right)=z_{0}=\left[\begin{array}{c}x_{0} \\ 0\end{array}\right]$ so that

$$
\begin{aligned}
& \left(\prod_{j=1}^{k}\left[\begin{array}{ll}
I & 0 \\
0 & 0
\end{array}\right] e^{\Lambda h(j)}\right)\left[\begin{array}{c}
x_{0} \\
0
\end{array}\right] \\
& =\left[\begin{array}{cc}
\left(\prod_{j=1}^{k} S_{j}\right) x_{0} & 0 \\
0 & 0
\end{array}\right] \\
& =\left(\prod_{j=1}^{k}\left[\begin{array}{ll}
I & 0 \\
0 & 0
\end{array}\right] e^{\Lambda h(j)}\left[\begin{array}{ll}
I & 0 \\
0 & 0
\end{array}\right]\right)\left[\begin{array}{l}
x_{0} \\
0
\end{array}\right] .
\end{aligned}
$$

In view of (5), it is clear that we can represent the system response as in (2).

Note that in (2) the response is given by a product of matrices that share similar structure but are in general different. All $M(j)$ are the same in the case where the update times $h(k)$ are equal. This constant $h$ case was studied in [11], [12], and [14] where necessary and sufficient conditions for stability were derived. In the present case of varying $h(k)$, stability cannot be guaranteed even if all matrices in the product have their eigenvalues inside the unit circle. To provide some insight into the stability for the constant update case before we derive conditions for time varying update times in the next section, we recall the results obtained for constant $h$ [14]. The proof for Theorem 1 may be found in [14].

Theorem 1: The system described by (1) with $h(k)=h$ a positive constant, is globally exponentially stable around the solution $z=\left[\begin{array}{ll}x^{T} & e^{T}\end{array}\right]^{T}=\left[\begin{array}{ll}0 & 0\end{array}\right]^{T}$ if and only if the eigenvalues of

$$
M=\left[\begin{array}{ll}
I & 0 \\
0 & 0
\end{array}\right] e^{\Lambda h}\left[\begin{array}{ll}
I & 0 \\
0 & 0
\end{array}\right]
$$

are strictly inside the unit circle.

It can be shown [14] that the upper left block of the test matrix $M$, which may contain nonzero entries, is given by

$$
\begin{gathered}
N=\mathrm{e}^{(\hat{A}+\hat{B} K)^{T} h}+\Delta(h)^{T} \\
\text { where } \Delta(h)=\int_{0}^{h} e^{A(h-\tau)}(\tilde{A}+\tilde{B} K) e^{(\hat{A}+\hat{B} K) \tau} d \tau .
\end{gathered}
$$


Note that the structure of $N$ clearly reveals the dependency on the update time $h$, the stable compensated model eigenvalues, and the difference between the plant and the model. Here, $\Delta(h)$ can be treated as a perturbation over the eigenvalues of the compensated plant model. Note also that $\Delta(h)$ can be efficiently computed using

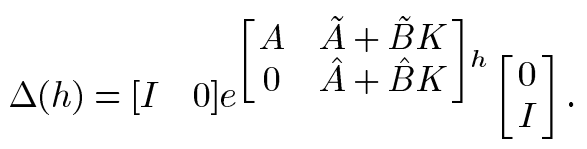

Conservative versions of Theorem 1 can be obtained by bounding (6) with an appropriate norm so to isolate the modeling errors from the model description. Then, however, these conditions will be sufficient only and typically more conservative. The results obtained in Theorem 1 can be applied as shown in the next example.

Example 1: We will use a nominal model and a plant description where two entries in the $A$ matrix can vary within a certain interval.

$$
\begin{aligned}
& \text { model : } \quad \hat{A}=\left[\begin{array}{ll}
0 & 1 \\
0 & 0
\end{array}\right] \quad \hat{B}=\left[\begin{array}{l}
0 \\
1
\end{array}\right] \\
& \text { plant : } \quad A=\left[\begin{array}{cc}
0 & 1+\tilde{a}_{12} \\
0+\tilde{a}_{21} & 0
\end{array}\right] \quad B=\left[\begin{array}{l}
0 \\
1
\end{array}\right] \\
& \text { with } \quad \tilde{a}_{12}=[-0.5,0.5] \quad \tilde{a}_{21}=[-0.5,0.5] \\
& \text { controller : } \quad K=[-1,-2] \text {. }
\end{aligned}
$$

Fig. 2 shows the contour levels representing the maximum eigenvalue magnitude for the test matrix $M$ as a function of the values of the entries $(1,2)$ and $(2,1)$ of the plant matrix $A$. The update time $h$ was $2.5 \mathrm{~s}$ It is easy to isolate the stable and unstable regions in the uncertainty parameter plane. The stable region lies in between the two contours labeled one. Note that in the case of more than two uncertain parameters a search algorithm may be used to determine the stability region. The necessary and sufficient results of Theorem 1 can be used to determine the stability of the system for any point in the parameter space.

\section{LYAPUNOV STABILITY}

Let us return now to the time varying case of update times $h(k)$. The stability criterion derived in this section is the strongest and most conservative stability criterion of this paper. It is based on the well-known Lyapunov second method for determining the stability of a system. We will assume that the properties of $h(k)$ are unknown but $h(k)$ is contained within some interval. This criterion is not stochastic but provides a first approach to stability for time-varying transmission times NCS.

Definition 1: The equilibrium $z=0$ of a system described by $\dot{z}=f(t, z)$ with initial condition $z\left(t_{0}\right)=z_{0}$ is Lyapunov asymptotically stable at large (or globally) if for any $\varepsilon>0$ there exists $\beta>0$ such that the solution $\dot{z}=f(t, z)$ satisfies

$$
\left\|z\left(t, z_{0}, t_{0}\right)\right\|<\varepsilon \quad \forall t>t_{0} \text {, and } \lim _{t \rightarrow \infty}\left\|z\left(t, z_{0}, t_{0}\right)\right\|=0
$$

whenever $\left\|z_{0}\right\|<\beta$.

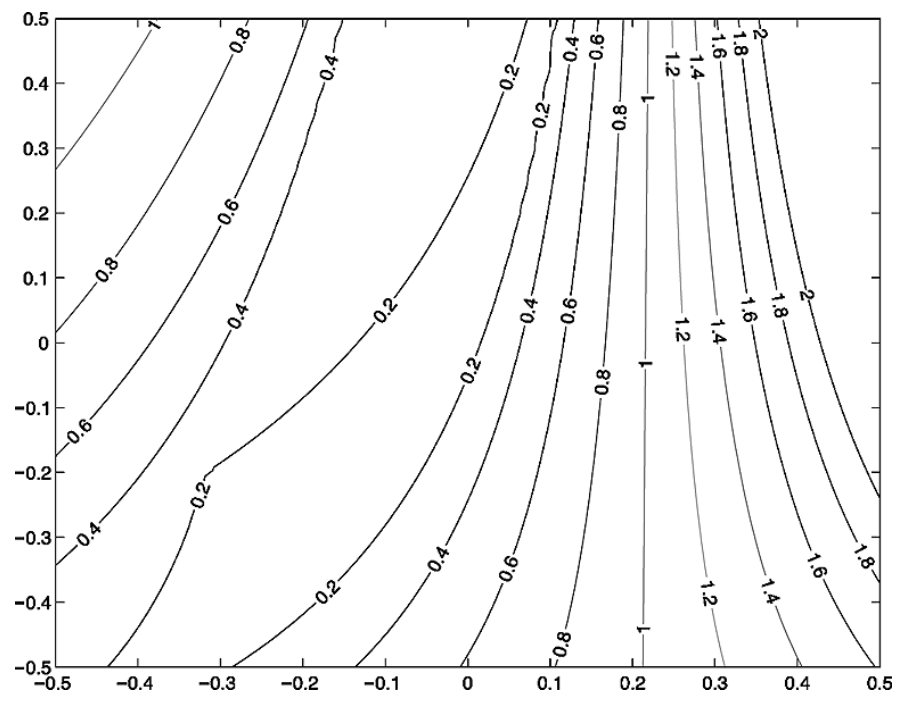

Fig. 2. Maximum eigenvalue magnitude versus model error.

Theorem 2: The system described by (2) is Lyapunov asymptotically stable for $h \in\left[h_{\min }, h_{\max }\right]$ if there exists a symmetric positive-definite matrix $X$ such that $X-M X M^{T}=Q$ is positive definite for all $h \in\left[h_{\min }, h_{\max }\right]$, where

$$
M=\left[\begin{array}{ll}
I & 0 \\
0 & 0
\end{array}\right] e^{\Lambda h}\left[\begin{array}{ll}
I & 0 \\
0 & 0
\end{array}\right] .
$$

Proof: Note that the output norm can be bounded by

$$
\begin{aligned}
& \left\|e^{\Lambda\left(t-t_{k}\right)}\left(\prod_{j=1}^{k} M(j)\right) z_{0}\right\| \\
& \leq\left\|e^{\Lambda\left(t-t_{k}\right)}\right\| \cdot\left\|\prod_{j=1}^{k} M(j)\right\| \cdot\left\|z_{0}\right\| \\
& \leq e^{\bar{\sigma}(\Lambda) h_{\max }} \cdot\left\|\prod_{j=1}^{k} M(j)\right\| \cdot\left\|z_{0}\right\| .
\end{aligned}
$$

That is, since $e^{\Lambda\left(t-t_{k}\right)}$ has finite growth rate, it will grow for at most until $h_{\max }$. Then, convergence of the product of matrices $M(j)$ to zero ensures the stability of the system. Such convergence to zero is guaranteed by the existence of a symmetric positive-definite matrix $X$ in the Lyapunov equation.

Theorem 2 may be used to derive an interval $\left[h_{\min }, h_{\max }\right]$ for $h$ for which stability is guaranteed. It is clear that the range for $h$, that is the interval $\left[h_{\min }, h_{\max }\right]$, will vary with the choice of $X$. Another observation is that the interval obtained this way will always be contained in the set of constant update times for which the system is stable (as derived using Theorem 1). That is, an update time contained in the interval $\left[h_{\min }, h_{\max }\right]$ will always be a stable constant update time.

Several ways of obtaining the values for $h_{\min }$ and $h_{\max }$ can be used. One is to first fix the value of $Q$, obtain the solution $X$ of the Lyapunov equation in Theorem 2 for a value of $h$ known to be stable. Then, using this value of $X$, the expression $X-$ $M X M^{T}$ can be evaluated for positive definiteness. This can be 
repeated for all the values of $h$ known to stabilize the system to obtain the widest interval $\left[h_{\min }, h_{\max }\right]$.

Example: We use an unstable plant, the double integrator where

$$
A=\left[\begin{array}{ll}
0 & 1 \\
0 & 0
\end{array}\right] \quad B=\left[\begin{array}{l}
1 \\
0
\end{array}\right]
$$

For our plant model, we chose

$$
\dot{\hat{x}}=\hat{A} \hat{x}+\hat{B} u \quad \hat{A}=\left[\begin{array}{ll}
0 & 0 \\
0 & 0
\end{array}\right] \quad \hat{B}=\left[\begin{array}{l}
0 \\
0
\end{array}\right]
$$

which behaves as a zero order hold device, since it maintains the value of the state vector and control signal constant until the next update time. Our feedback law is given by $u=K x$ with $K=[-1-2]$. When the update times are constant, stability can be guaranteed if and only if the eigenvalues of the test matrix $M$ are inside of the unit circle [11], [12]. It can be verified that, for stability, the set of constant update times for our example is $[0,1) \mathrm{s}$. We now use Theorem 2 to find an interval of time varying update times for which the NCS will remain stable. Note that the update times can vary along this interval. We will set a nominal update time of $0.5 \mathrm{~s}$ and set $Q$ to be the identity matrix; then by solving the Lyapunov equation given in Theorem 2 we can find a positive definite nominal $X$. We then obtain the stability interval by searching for update times around 0.5 seconds, for which the $Q$ in the Lyapunov equation obtained with the nominal $X$ is positive definite. Fig. 3 shows the plot of the minimum eigenvalue of $Q$ as a function of the update time, from which it can be seen that the stability interval is now $\left[h_{\min }, h_{\max }\right]=[0,0.85]$. Note that it has been shown [11] that for constant $h$ that the system remains stable for any $h<1$. Of course, by using different nominal update times and nominal $Q$ 's less conservative results may be obtained.

\section{Almost Sure or Probability-1 Asymptotic Stability}

We will use the definition of almost sure asymptotic stability [8] that provides a stability criterion based on the sample path. This stability definition resembles more the deterministic stability definition [9], and is of practical importance. Since the stability condition has been relaxed, we expect to see less conservative results than those obtained using the Lyapunov stability considered in the previous section. We now define almost sure or probability-1 asymptotic stability.

Definition 2: The equilibrium $z=0$ of a system described by $\dot{z}=f(t, z)$ with initial condition $z\left(t_{0}\right)=z_{0}$ is almost sure (or with probability-1) asymptotically stable at large (or globally) if for any $\beta>0$ and $\varepsilon>0$ the solution of $\dot{z}=f(t, z)$ satisfies

$$
\lim _{\delta \rightarrow \infty} P\left\{\sup _{t \geq \delta}\left\|z\left(t, z_{0}, t_{0}\right)\right\|>\varepsilon\right\}=0
$$

whenever $\left\|z_{0}\right\|<\beta$.

This definition is similar to the one presented for deterministic systems in Definition 1. We will examine the conditions under which the full-state feedback continuous networked system in Fig. 1 is stable.

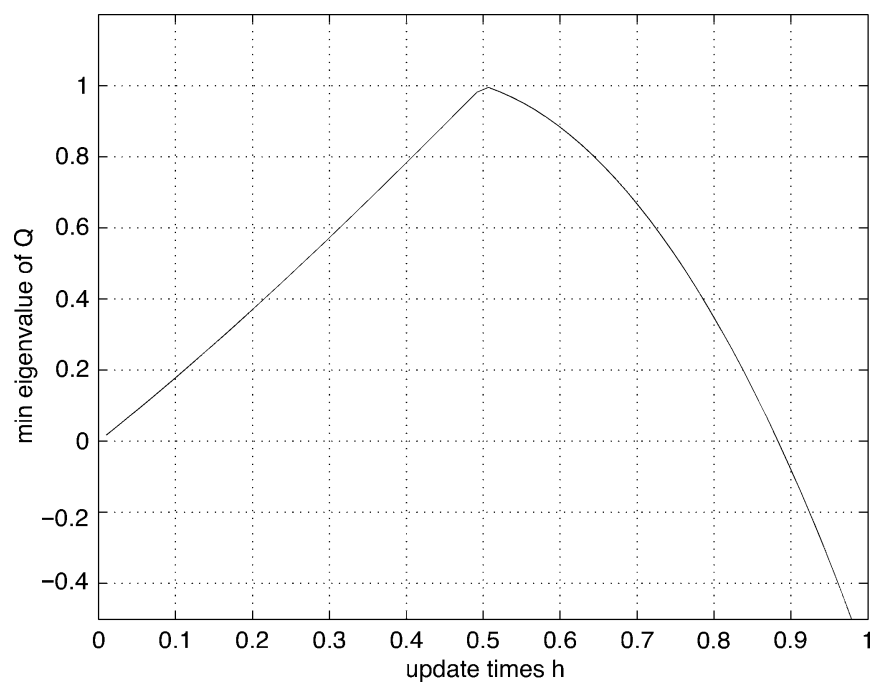

Fig. 3. Minimum eigenvalue of $Q$ as a function of the update time $h$.

\section{A. MB-NCS With Independent Identically Distributed Transmission Times}

Here, we will assume that the update times $h(k)$ are independent identically distributed (iid) with probability distribution function $F(h)$. We now present the conditions under which the system described by (2) with iid update times is asymptotically stable with probability-1. We will use a technique similar to lifting [2] to obtain a discrete linear time-invariant representation of the system. It can be observed that the system will be described by

$$
\xi_{k+1}=\Omega_{k} \xi_{k}, \quad \text { with } \xi_{k} \in L_{2 e} \text { and } \xi_{k}=z\left(t+t_{k}\right), t \in\left[0, h_{k}\right) \text {. }
$$

Here, $L_{2 e}$ stands for the extended $L_{2}$. It is easy to obtain from (2) the operator $\Omega_{k}$ as

$$
\left(\Omega_{k} \nu\right)(t)=e^{\Lambda t}\left[\begin{array}{ll}
I & 0 \\
0 & 0
\end{array}\right] \int_{0}^{h(k)} \delta(\tau-h(k)) \nu(\tau) d \tau .
$$

Now, we can restate the definition on almost sure stability or probability-1 stability given in Definition 2 to better fit the equivalent system representation (11).

Definition 3: The system represented by (11) is almost sure stable or stable with probability- 1 if for any $\beta>0$ and $\varepsilon>0$ the solution of $\xi_{k+1}=\Omega_{k} \xi_{k}$ satisfies

$$
\lim _{\tilde{\delta} \rightarrow \infty} P\left\{\sup _{k \geq \tilde{\delta}}\left\|\xi_{k}\left(t_{0}, z_{0}\right)\right\|_{2,\left[0, t_{k}\right]}>\varepsilon\right\}=0
$$

whenever $\left\|z_{0}\right\|<\beta$. Where the norm $\|\cdot\|_{2,[0, h(k)]}$ is given by $\left\|\xi_{k}\right\|_{2,[0, h(k)]}=\left(\int_{0}^{h(k)}\left\|\xi_{k}(\tau)\right\|^{2} d \tau\right)^{1 / 2}$.

This definition allows us to study almost sure stability of systems such as (11) when the probability distribution function for update times has infinite support. Based on this definition the following result can now be shown.

Theorem 3: The system described by (2), with update times $h(j)$ independent identically distributed random variable with probability distribution $F(h)$ is globally almost sure (or with 
probability-1) asymptotically stable around the solution $z=$ $\left[\begin{array}{ll}x^{T} & e^{T}\end{array}\right]^{T}=\left[\begin{array}{ll}0 & 0\end{array}\right]^{T}$ if $N=E\left[\left(e^{2 \bar{\sigma}(\Lambda) h}-1\right)^{1 / 2}\right]<\infty$ and the expected value of the maximum singular value of the test matrix $M, E[\|M\|]=E\left[\bar{\sigma}_{M}\right]$, is strictly less than one, where $M=\left[\begin{array}{ll}I & 0 \\ 0 & 0\end{array}\right] e^{\Lambda h}\left[\begin{array}{ll}I & 0 \\ 0 & 0\end{array}\right]$.

Proof: Assume that the supremum of the norm bracketed is achieved at $k^{*} \geq \tilde{\delta}$, that is $\sup _{\tilde{\delta}}\left\|\xi_{k}\right\|=\left\|\xi_{k^{*}}\right\|$. So, now we $k>\tilde{\delta}$.

can use Chebyshev bound for positive random variables [20] to bound the probability in our definition

$$
P\left\{\sup _{k \geq \tilde{\delta}}\left\|\xi_{k}\right\|>\varepsilon\right\}=P\left\{\left\|\xi_{k^{*}}\right\|>\varepsilon\right\} \leq \frac{E\left[\left\|\xi_{k^{*}}\right\|\right]}{\varepsilon} .
$$

Using (2) and basic norm properties, we proceed to bound the expectation on the right-hand side of

$$
\begin{aligned}
E\left[\left(\int_{0}^{h\left(k^{*}\right)}\left\|\xi_{k^{*}}(\tau)\right\|^{2} d \tau\right)^{\frac{1}{2}}\right] \\
\left.\leq E\left[\int_{0}^{h\left(k^{*}\right)}\left\|e^{\Lambda \tau}\right\|^{2}\left\|\prod_{j=1}^{k^{*}-1} M(j)\right\|^{2}\left\|z_{0}\right\|^{2} d \tau\right)^{\frac{1}{2}}\right] \\
\leq E \\
{\left[\left(\int_{0}^{h\left(k^{*}\right)}\left(e^{\left.\bar{\sigma}(\Lambda) \tau)^{2} d \tau\right)^{\frac{1}{2}} \| k^{*}-1}\left\|\prod_{j=1} M(j)\right\| z_{0} \|\right]\right.\right.} \\
=E\left[\left(\int_{0}^{h\left(k^{*}\right)}\left(e^{\bar{\sigma}(\Lambda) \tau)^{2} d \tau}\right)^{\frac{1}{2}}\right]\right. \\
\times E\left[\left\|\prod_{j=1}^{k^{*}-1} M(j)\right\| z_{0} \| .\right.
\end{aligned}
$$

The last equation follows from the independence of the update times $h(j)$. Analyzing the first term on the last equality we see that is bounded for the trivial case where $\Lambda=0$. When $\Lambda \neq 0$, the integral can be solved, and can be showed to be equal to $\sqrt{1 /(2 \bar{\sigma}(\Lambda))} E\left[\left(e^{2 \bar{\sigma}(\Lambda) h\left(k^{*}\right)}-1\right)^{1 / 2}\right]$ which by assumption is bounded. The second term can also be bounded by using the independence property of the update times $h(j)$

$E\left[\left\|\prod_{j=1}^{k^{*}-1} M(j)\right\|\right] \leq E\left[\prod_{j=1}^{k^{*}-1}\|M(j)\|\right]=(E[\|M\|])^{k^{*}-1}$.
We can now evaluate the limit over the expression obtained, as shown in (17) at the bottom of the page. It is obvious that the right-hand side of the expression will be identically zero (note that $\left.k^{*} \geq \tilde{\delta}\right)$ if the average maximum singular value $E\left[\bar{\sigma}_{M}\right]=$ $E[\|M\|]<1$.

Note that the condition may give conservative results if applied directly to the test matrix. To avoid this problem and make the condition tighter we may apply a similarity transformation over the test matrix $M$. The condition on the matrix $N$ ensures that the probability distribution function for the update times $F(h)$ assigns smaller occurrence probabilities to increasingly long update times, that is $F(h)$ decays rapidly. In particular, we observe that $N$ can always be bounded if there exists $h_{m}$ such that $F(h)=0$ for $h$ larger than $h_{m}$. We can also bound the expression inside the expectation to obtain $E\left[\left(e^{2 \sigma(\Lambda) h}-1\right)^{1 / 2}\right]<$ $E\left[e^{\sigma(\Lambda) h}\right]$ and formulate the following corollary.

Corollary 1: The system described by (2), with update times $h(j)$ that are independent identically distributed random variable with probability distribution $F(h)$ is globally almost sure (or with probability-1) asymptotically stable around the solution $z=\left[\begin{array}{ll}x^{T} & e^{T}\end{array}\right]^{T}=\left[\begin{array}{ll}0 & 0\end{array}\right]^{T}$ if $T=E\left[e^{\sigma(\Lambda) h}\right]<\infty$ and the expected value of the maximum singular value of the test matrix $M, E[\|M\|]=E\left[\bar{\sigma}_{M}\right]$, is strictly less than one, where

$$
M=\left[\begin{array}{ll}
I & 0 \\
0 & 0
\end{array}\right] e^{\Lambda h} \quad\left[\begin{array}{ll}
I & 0 \\
0 & 0
\end{array}\right] .
$$

Note that Corollary 1 condition $T=E\left[e^{\sigma(\Lambda) h}\right]<\infty$ is automatically satisfied if the probability distribution function $F(h)$ does not has infinite support. It otherwise indicates that $F(h)$ should roll off fast enough as to counteract the growth of $M$ 's maximum singular value as $h$ increases.

Example 3: We use the same unstable double integrator plant used in Example 2. We now assume that $h(k)$ is a random variable with a uniform probability distribution function $U\left(0.5, h_{\max }\right)$. The plot of the expected maximum singular value of a similarity transformation of the original test matrix $M$ is shown in Fig. 4. The similarity transformation used here was one that diagonalizes the matrix $M$ for $h=1$. We see that the maximum value for $h_{\max }$ is around $1.3 \mathrm{~s}$ (maximum constant update time for stability is $h=1 \mathrm{~s}$.) So we see that, the double integrator with uniformly distributed update time between 0.5 and $1.3 \mathrm{~s}$ is stable, while the same system but with a constant update time of $1 \mathrm{~s}$ is unstable [11]. This also represents an improvement over the result that we may have obtained by using the previously discussed Lyapunov Stability condition in which the maximum update time obtainable would have been less than $1 \mathrm{~s}$. We can use the expected value of the

$$
\begin{aligned}
\lim _{\tilde{\delta} \rightarrow \infty} P\left\{\sup _{k \geq \tilde{\delta}}\left\|\xi_{k}\right\|>\varepsilon\right\} & \leq \lim _{\tilde{\delta} \rightarrow \infty} \frac{E\left[\left\|\xi_{k^{*}}\right\|\right]}{\varepsilon} \\
& \leq \lim _{\tilde{\delta} \rightarrow \infty} \frac{\sqrt{\frac{1}{2 \bar{\sigma}(\Lambda)}} E\left[\left(e^{2 \bar{\sigma}(\Lambda) h\left(k^{*}\right)}-1\right)^{\frac{1}{2}}\right](E[\|M\|])^{k^{*}-1}\left\|z_{0}\right\|}{\varepsilon} .
\end{aligned}
$$




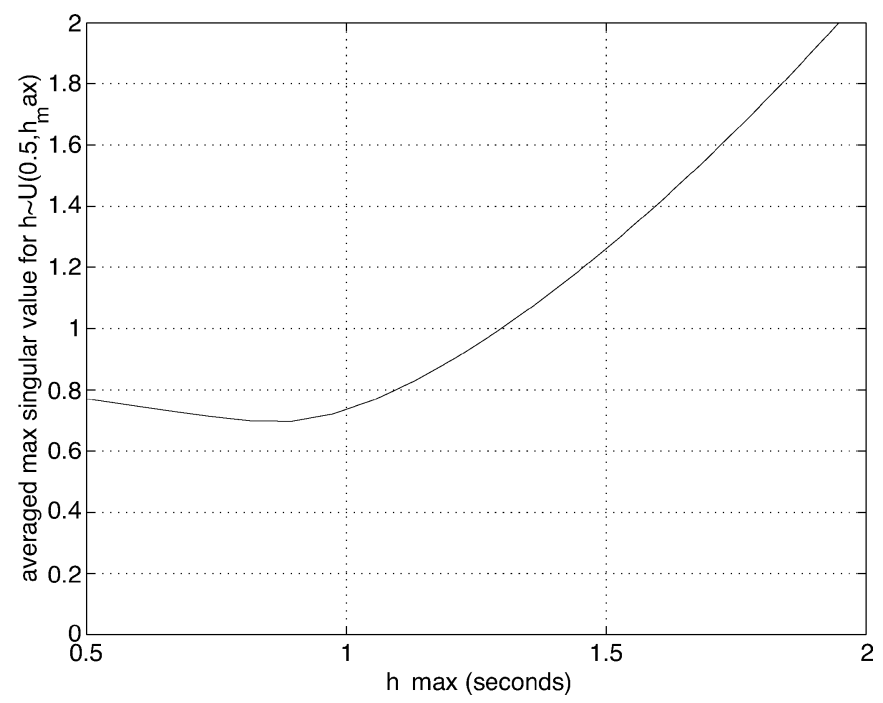

Fig. 4. Average maximum singular value for $\overline{\bar{\sigma}}_{M}=E[\|M\|]$ for $h \sim U\left(0.5, h_{\max }\right)$ as a function of $h_{\max }$, zero dynamics plant model.

spectral radius of the test matrix as a measure of the system performance: This measure is related to the contraction rate of the system. On a hyper-parallelogram with axis defined by the eigenvectors of $M$, the spectral radius will indicate the contraction rate of the hyper-parallelogram axis with the slowest contraction rate. Our performance measure is defined in (18), here $\rho(M)$ denotes the spectral radius of $M$. Since the plant is continuous, the update interval $h$ is also included in the expectation to give consistency to the performance measure

$$
P_{R}=-E\left[\frac{\ln (\rho(M))}{h}\right] \text {. }
$$

In Fig. 5, we plot the performance measure as a function of the maximum update time for the system described in the example. Note that the performance measure is almost the same for update times with uniform probability distribution $U\left(0.5, h_{\max }\right)$ with, $h_{\max }$ up to $0.8 \mathrm{~s}$. The performance measure for a fixed update time of $0.5 \mathrm{~s}$ is 0.727 while the measure for update times with uniform probability distribution $U(0.5,0.8)$ is about 0.713 . It is clear that having an update time that can vary among an interval is better that a fixed update time in terms of implementation: Soft real time constraints are easier to enforce than hard realtime constraints.

The advantage of using a model-based approach resides in its ability to reduce the amount of bandwidth required. The previous example shows stability conditions for a model that represents a zero order hold, that is, the control value is kept constant until the next update time. We will now show the same plots for a model that better resembles the plant, this was done by randomly perturbing the plant matrices. The plant model matrices are

$$
\hat{A}=\left[\begin{array}{cc}
0.0844 & 0.9353 \\
0.0476 & -0.0189
\end{array}\right] \quad \hat{B}=\left[\begin{array}{l}
0.0871 \\
1.0834
\end{array}\right] .
$$

Fig. 6 shows that stability is maintained for update times that have uniform distribution with a max update time of $5.5 \mathrm{~s}$. Fig. 7 shows that the performance measure is higher than in the case

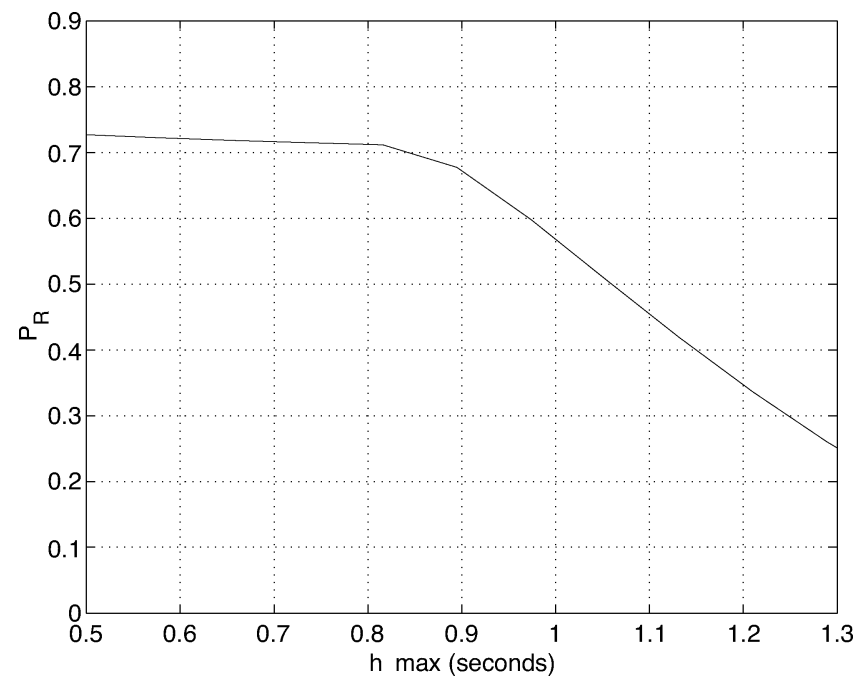

Fig. 5. Performance measure for $h \sim U\left(0.5, h_{\max }\right)$ as a function of $h_{\max }$, zero dynamics plant model.

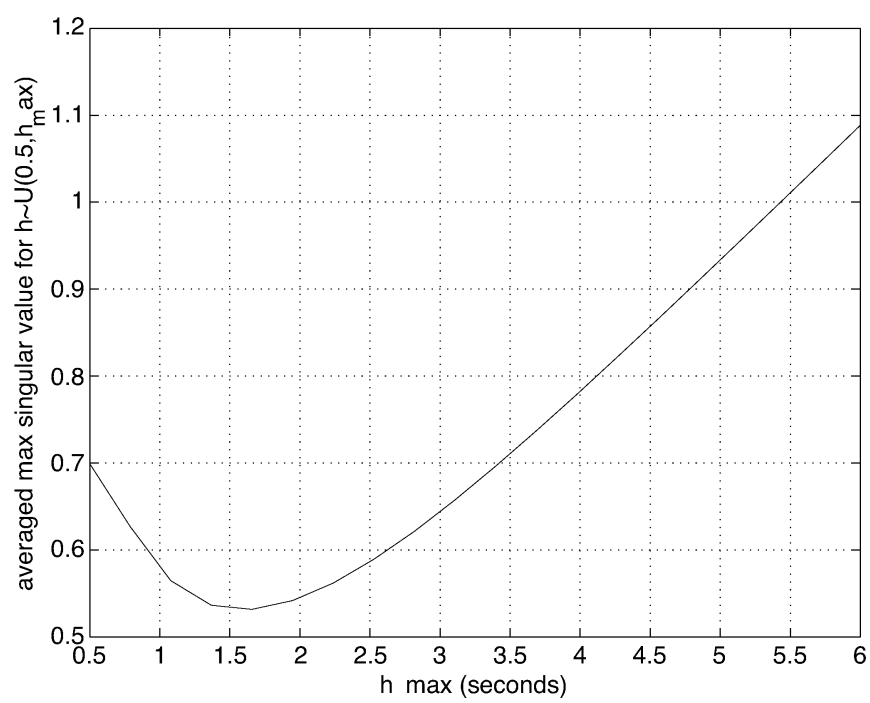

Fig. 6. Average maximum singular value for $\overline{\bar{\sigma}}_{M}=E[\|M\|]$ for $h \sim U\left(0.5, h_{\max }\right)$ as a function of $h_{\max }$, improved plant model.

of the zero-order hold model. This shows that improved knowledge over the plant dynamics can translate into a significant improvement in terms of stability and performance measure.

\section{B. MB-NCS With Markov Chain-Driven Transmission Times}

In certain cases, it is appropriate to represent the dynamics of the update times as driven by a Markov chain. A good example of this is when the network experiences traffic congestion or has queues for message forwarding. We now present a stability criterion for the model-based control system in which the update times $h(k)$ are driven by a finite state Markov chain. Assume that the update times can take a value from a finite set

$$
h(k) \in\left\{h_{1}, h_{2}, \ldots, h_{N}\right\} \text { and } h_{i} \neq \infty \quad \forall i \in[1, N] .
$$

Let us represent the Markov chain process by $\left\{\omega_{k}\right\}$ with state-space $\{1,2, \ldots, N\}$ and transition probability matrix $\Gamma$ and $N \times N$ matrix with elements $p_{i, j}$ and initial state probability distribution $\Pi_{0}=\left[\begin{array}{llll}\pi_{1} & \pi_{2} & \ldots & \pi_{N}\end{array}\right]^{T}$. The transition probability 


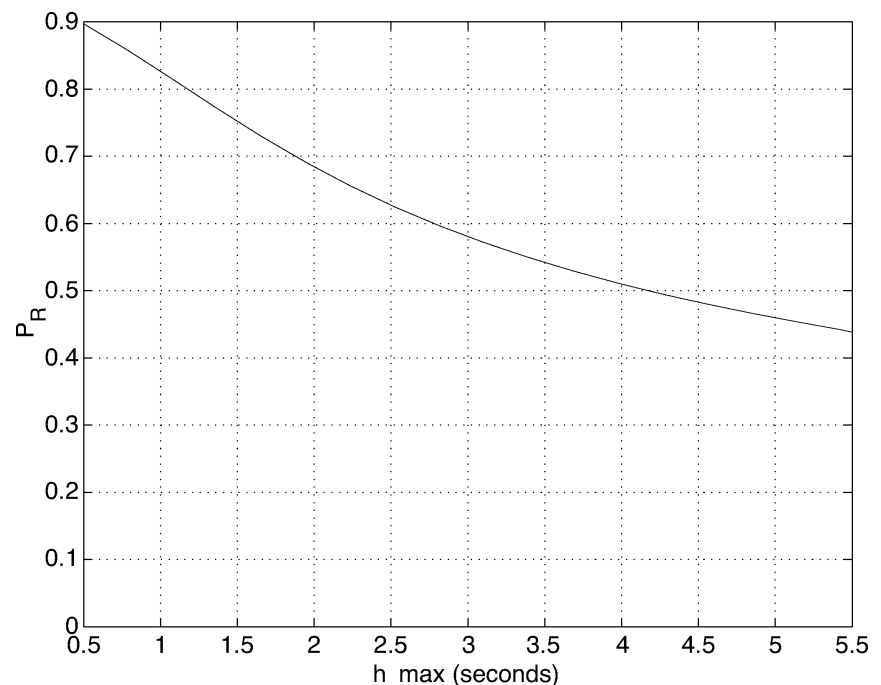

Fig. 7. Performance measure for $h \sim U\left(0.5, h_{\max }\right)$ as a function of $h_{\max }$, improved plant model.

matrix entries are defined as $p_{i, j}=\mathrm{P}\left\{\omega_{k+1}=j \mid \omega_{k}=i\right\}$. We can now represent the update times more appropriately as $h(k)=h_{\omega_{k}}$. sufficient condition for the almost sure stability of the system under Markovian jumps is given in the following theorem.

Theorem 4: The system described by (2), with update times $h(k)=h_{\omega_{k}} \neq \infty$ driven by a finite state Markov chain $\left\{\omega_{k}\right\}$ with state-space $\{1,2, \ldots, N\}$ and transition probability matrix $\Gamma$ with elements $p_{i, j}$ and initial state probability distribution $\Pi_{0}=\left[\begin{array}{lllll}\pi_{1} & \pi_{2} & \ldots & \pi_{N}\end{array}\right]^{T}$ is globally almost sure asymptotically stable around the solution $z=\left[\begin{array}{ll}x^{T} & e^{T}\end{array}\right]^{T}\left[\begin{array}{ll}0 & 0\end{array}\right]^{T}$ if the matrix $T$ has all its eigenvalues inside of the unit circle, where

$$
\begin{aligned}
T= & {\left[\begin{array}{cccc}
\left\|\left.M\right|_{h=h_{1}}\right\| & 0 & \cdots & 0 \\
0 & \left\|\left.M\right|_{h=h_{2}}\right\| & \cdots & 0 \\
\vdots & \vdots & \ddots & \vdots \\
0 & 0 & \cdots & \left\|\left.M\right|_{h=h_{N}}\right\|
\end{array}\right] } \\
& \times \Gamma^{T}
\end{aligned}
$$

and $\left.M\right|_{h=h_{i}}=\left[\begin{array}{ll}I & 0 \\ 0 & 0\end{array}\right] e^{\Lambda h_{i}}\left[\begin{array}{ll}I & 0 \\ 0 & 0\end{array}\right]$.

Proof: It is clear that since the Markov chain has a finite number of states, the update times are bounded. Then, using the same argument in Theorem 2 we can bound the output by

$$
\begin{aligned}
& \left\|e^{\Lambda\left(t-t_{k}\right)}\left(\prod_{j=1}^{k} M(j)\right) z_{0}\right\| \\
& \leq\left\|e^{\Lambda\left(t-t_{k}\right)}\right\| \cdot\left\|\prod_{j=1}^{k} M(j)\right\| \cdot\left\|z_{0}\right\| \\
& \leq e^{\bar{\sigma}(\Lambda) h_{\max }} \cdot\left\|\prod_{j=1}^{k} M(j)\right\| \cdot\left\|z_{0}\right\| .
\end{aligned}
$$

Therefore, we can ensure stability by studying the convergence of the term $\left\|\prod_{j=1}^{k} M(j)\right\|$. We can now use a similar procedure as the one used for Theorem 3. For almost sure stability, we will require that

$$
\lim _{\tilde{\delta} \rightarrow \infty} P\left\{\sup _{k \geq \tilde{\delta}}\left\|\prod_{j=1}^{k} M(j)\right\|>\varepsilon\right\}=0 .
$$

We will assume that the supremum of the norm bracketed is achieved at $k^{*} \geq \tilde{\delta}$. Using the Chevyshev inequality, we obtain

$$
\begin{gathered}
P\left\{\sup _{k \geq \tilde{\delta}}\left\|\prod_{j=1}^{k} M(j)\right\|>\varepsilon\right\} \\
=P\left\{\left\|\prod_{j=1}^{k_{*}} M(j)\right\|>\varepsilon\right\} \\
\leq \frac{E\left[\left\|\prod_{j=1}^{k_{*}} M(j)\right\|\right]}{\leq} \frac{E\left[\left\|\prod_{j=1}^{k_{*}}\right\| M(j)\|\|\right]}{\varepsilon} .
\end{gathered}
$$

Evaluating the expectation yields

$$
\begin{aligned}
& E\left[\prod_{j=1}^{k_{*}}\|M(j)\|\right] \\
& =\sum_{\forall\left[i_{0}, i_{1}, \ldots, i_{k_{*}-1}\right]}\left\{( \prod _ { j = 1 } ^ { k _ { * } } \| M ( j ) \| ) P \left\{\left[\omega_{0}=i_{0}, \omega_{1}=i_{1}, \ldots,\right.\right.\right. \\
& \left.\left.\left.\omega_{k_{*}-1}=i_{k_{*}-1}\right]\right\}\right\} \\
& =\sum_{\forall\left[i_{0}, i_{1}, \ldots, i_{k *-1}\right]}\left(\prod_{j=1}^{k_{*}}\|M(j)\| p_{i_{j-1}, i_{j-2}}\right)\|M(1)\| \pi_{i_{0}} \\
& =[1,1, \ldots, 1] \\
& \times\left(\left[\begin{array}{cccc}
\left\|\left.M\right|_{h=h_{1}}\right\| & 0 & \cdots & 0 \\
0 & \left\|\left.M\right|_{h=h_{2}}\right\| & \cdots & 0 \\
\vdots & \vdots & \ddots & \vdots \\
0 & 0 & \cdots & \left\|\left.M\right|_{h=h_{N}}\right\|
\end{array}\right] \Gamma^{T}\right)^{k} \\
& \times\left[\begin{array}{l}
\left\|\left.M\right|_{h=h_{1}}\right\| \pi_{1} \\
\left\|\left.M\right|_{h=h_{2}}\right\| \pi_{1} \\
\cdots \\
\left\|\left.M\right|_{h=h_{N}}\right\| \pi_{1}
\end{array}\right]
\end{aligned}
$$

where each $i_{n} \in\{1,2, \ldots, N\}$. Therefore, the right-hand side of (23) will converge to zero if $T$ has all its eigenvalues inside the unit circle where

$$
T=\left[\begin{array}{cccc}
\left\|\left.M\right|_{h=h_{1}}\right\| & 0 & \cdots & 0 \\
0 & \left\|\left.M\right|_{h=h_{2}}\right\| & \cdots & 0 \\
\vdots & \vdots & \ddots & \vdots \\
0 & 0 & \cdots & \left\|\left.M\right|_{h=h_{N}}\right\|
\end{array}\right] \Gamma^{T} .
$$


If $\Gamma$ is irreducible, it follows that, since $\|M\|$ is nonnegative, $T$ is also irreducible. Then it can be shown using the Perron-Frobenius theorem as in [4], that T's maximum magnitude eigenvalue is real and sometimes referred to as the Perron-Frobenius eigenvalue.

\section{MEAN-SQuare or Quadratic Asymptotic Stability}

We now define a different type of stability, namely meansquare asymptotic stability.

Definition 4: The equilibrium $z=0$ of a system described by $\dot{z}=f(t, z)$ with initial condition $z\left(t_{0}\right)=z_{0}$ is mean-square stable asymptotically stable at large (or globally) if the solution of $\dot{z}=f(t, z)$ satisfies

$$
\lim _{t \rightarrow \infty} E\left[\left\|z\left(t, z_{0}, t_{0}\right)\right\|^{2}\right]=0 .
$$

A system that is mean-square stable will have the expectation of system states converging to zero with time in the mean-square sense. This definition of stability is attractive since many optimal control problems use the squared norm in their formulations. We will study the two cases studied in the previous section under this new stability criterion.

\section{A. MB-NCS With Independent Identically Distributed Transmission Times}

We present the conditions under which the networked control system described in (2) is mean-square stable, we also discuss how these conditions relate to the ones for probability-1 stability.

Theorem 5: The system described by (2), with update times $h(j)$ independent identically distributed random variable with probability distribution $F(h)$ is globally mean-square asymptotically stable around the solution $z=\left[\begin{array}{ll}0 & 0\end{array}\right]^{T}$ if $K=E\left[\left(e^{\bar{\sigma}(\Lambda) h}\right)^{2}\right]<\infty$ and the maximum singular value of the expected value of $M^{T} M,\left\|E\left[M^{T} M\right]\right\|=\bar{\sigma}\left(E\left[M^{T} M\right]\right)$, is strictly less than one, where

$$
M=\left[\begin{array}{ll}
I & 0 \\
0 & 0
\end{array}\right] e^{\Lambda h} \quad\left[\begin{array}{ll}
I & 0 \\
0 & 0
\end{array}\right]
$$

Proof: Let us start by evaluating the expectation of the squared norm of the output of the system described by (2)

$$
\begin{aligned}
E & {\left[\left\|e^{\Lambda\left(t-t_{k}\right)} G(k) z_{0}\right\|^{2}\right] } \\
& =E\left[z_{0}^{T}(G(k))^{T}\left(e^{\Lambda\left(t-t_{k}\right)}\right)^{T} e^{\Lambda\left(t-t_{k}\right)} G(k) z_{0}\right] \\
& \leq E\left[\bar{\sigma}\left(\left(e^{\Lambda\left(t-t_{k}\right)}\right)^{T} e^{\Lambda\left(t-t_{k}\right)}\right) z_{0}^{T}(G(k))^{T} G(k) z_{0}\right] \\
& \leq E\left[\left(e^{\bar{\sigma}(\Lambda) h(k+1)}\right)^{2} z_{0}^{T}(G(k))^{T} G(k) z_{0}\right] \\
& \text { where } G(k)=\prod_{j=1}^{k} M(j) .
\end{aligned}
$$

Now that the expectation is all in terms of the update times, we can use the independently identically distributed property of the update times and the assumption that $K$ is bounded

$$
\begin{aligned}
& E\left[\left(e^{\bar{\sigma}(\Lambda) h(k+1)}\right)^{2} z_{0}^{T}(G(k))^{T} G(k) z_{0}\right] \\
& \quad=K z_{0}^{T} E\left[(G(k-1))^{T} M(k)^{T} M(k) G(k-1)\right] z_{0} \\
& \quad=K z_{0}^{T} E\left[(G(k-1))^{T} E\left[M^{T} M\right] G(k-1)\right] z_{0} \\
& \quad \leq K \bar{\sigma}\left(E\left[M^{T} M\right]\right) z_{0}^{T} E\left[(G(k-1))^{T} G(k-1)\right] z_{0} .
\end{aligned}
$$

We can repeat the last three steps recursively to finally obtain

$$
E\left[\left\|e^{\Lambda\left(t-t_{k}\right)} G(k) z_{0}\right\|^{2}\right] \leq K\left(\bar{\sigma}\left(E\left[M^{T} M\right]\right)\right)^{k} z_{0}^{T} z_{0} .
$$

So, now it is easy to see that if $\left\|E\left[M^{T} M\right]\right\|=\bar{\sigma}\left(E\left[M^{T} M\right]\right)<$ 1 then the limit of the expectation as time approaches infinity is zero, which concludes the proof.

Note the similarity between the conditions given by Theorems 3 and 5. In Theorem 3, we require the expectation of the maximum singular value of the test matrix to be less than one. For the stability in Theorem 5, the maximum singular value of the expectation of $M^{T} M$ should be less than one.

\section{B. MB-NCS With MarkovChain-Driven Transmission Times}

We now present a sufficient condition for the mean-square stability of the MB-NCS with Markov chain-driven update times.

Theorem 6: The system described by (2), with update times $h(k)=h_{\omega_{k}} \neq \infty$ driven by a finite state Markov chain $\left\{\omega_{k}\right\}$ with state-space $\{1,2, \ldots, N\}$ and transition probability matrix $\Gamma$ with elements $p_{i, j}$ is globally mean-square asymptotically stable around the solution $z=\left[\begin{array}{ll}x^{T} & e^{T}\end{array}\right]^{T}=\left[\begin{array}{ll}0 & 0\end{array}\right]^{T}$ if there exists positive-definite matrices $P(1), P(2), \ldots, P(N)$ such that $\left(\sum_{j=1}^{N} p_{i, j}\left(H(i)^{T} P(j) H(i)\right)-P(i)\right)<0 \forall i, j=1, \ldots, N$ with $H(i)=e^{\Lambda h_{i}}\left[\begin{array}{ll}I & 0 \\ 0 & 0\end{array}\right]$.

Proof: Using the same argument used in Theorem 4, since the update times are bounded, we can analyze the system's stability by sampling it at a certain time between each update time. For this, we evaluate the response of the system described by (2) at times $t_{k}^{-}$

$$
z\left(t_{k+1}^{-}\right)=e^{\Lambda h_{k+1}}\left[\begin{array}{ll}
I & 0 \\
0 & 0
\end{array}\right]\left(t_{k}^{-}\right) .
$$

Lets define $\varsigma(k)=z\left(t_{k-1}^{-}\right)$and $H\left(\omega_{k}\right)=e^{\Lambda h_{\omega_{k}}}\left[\begin{array}{ll}I & 0 \\ 0 & 0\end{array}\right]$. Now, we can represent the sampled networked control system as

$$
\varsigma(k+1)=H\left(\omega_{k}\right) \varsigma(k) .
$$

To ensure mean-square stability we will make use of a Lyapunov function of quadratic form and analyze the expected value of its 
difference between two consecutive samples. We will use the following Lyapunov function:

$$
V\left(\varsigma(k), \omega_{k}\right)=\varsigma(k)^{T} P\left(\omega_{k}\right) \varsigma(k)
$$

The expected value of the difference is

$$
\begin{aligned}
E[\Delta V \mid \varsigma, i] & \\
= & E\left[V\left(\varsigma(k+1), \omega_{k+1}\right)-V\left(\varsigma(k), \omega_{k}\right) \mid \varsigma(k)=\varsigma, \omega_{k}=i\right] \\
= & E\left[\varsigma(k+1)^{T} P\left(\omega_{k+1}\right) \varsigma(k+1) \mid \varsigma(k)=\varsigma, \omega_{k}=i\right] \\
& -\varsigma^{T} P(i) \varsigma \\
= & E\left[\varsigma^{T} H\left(\omega_{k}\right)^{T} P\left(\omega_{k+1}\right) H\left(\omega_{k}\right) \varsigma \mid \omega_{k}=i\right]-\varsigma^{T} P(i) \varsigma \\
= & \sum_{j=1}^{N} p_{i, j}\left(\varsigma^{T} H(i)^{T} P(j) H(i) \varsigma\right)-\varsigma^{T} P(i) \varsigma \\
= & \varsigma^{T}\left(\sum_{j=1}^{N} p_{i, j}\left(H(i)^{T} P(j) H(i)\right)-P(i)\right) \varsigma .
\end{aligned}
$$

From this last equality, is it obvious that to ensure mean-square stability we need to have

$$
\left(\sum_{j=1}^{N} p_{i, j}\left(H(i)^{T} P(j) H(i)\right)-P(i)\right)<0 .
$$

This type of stability criteria depend on our ability to find appropriate $P(i)$ matrices. Several other results in jump system stability [3], [5] can be extended to obtain other conditions on stability of networked control systems. Note though, that most of the results available in the literature deal with similar but not identical type of systems.

\section{CONCLUSION}

In this paper, three feedback network communication models were presented. The first one assumed unknown statistical properties of the update times and was analyzed using Lyapunov techniques rendering conservative deterministic stability conditions. The other two assumed known stochastic characteristics of the update time process: Independent identically distributed random process and finite-state Markov chain. Each stochastic model was analyzed using almost sure stability, and mean-square stability criteria.

It is clear from the examples presented that both types of stochastic stability studied enables larger update times range compared to the Lyapunov type of stability. The extended stability regions found using the stochastic stability criteria here described are possible thanks to the added information on the statistics of the update times. The stability results presented here address only some of the different stochastic stability criteria available in the literature. We consider that the insight provided can be used to determine conditions for other stability types as well. Note also that other techniques such as Lyapunov exponents can be used to analyze the problem.

The results presented here for state feedback MB-NCSs can be easily extended to other model-based control architectures such as output feedback, communication with delays, and discrete plants such as the ones studied in [14]. Other extensions such as the effect of quantizers, external disturbances, and the case of nonlinear plants are currently under investigation.

\section{REFERENCES}

[1] A. Bemporad and M. Morari, "Robust model predictive control: A survey," in Robustness in Identification and Control, A. Garulli, A. Tesi, and A. Vicino, Eds. New York: Springer-Verlag, 1999, pp. 207-226.

[2] T. Chen and B. Francis, Optimal Sampled-Data Control Systems, 2nd ed. London, U.K.: Springer-Verlag, 1996.

[3] O. L. V. Costa and M. D. Fragoso, "Stability results for discrete-time linear systems with Markovian jumping parameters," J. Math. Anal. Applicat., vol. 179, pp. 154-178, 1993.

[4] A. Dembo and O. Zeitouni, Large Deviation Techniques and Applications. New York: Springer-Verlag, 1998.

[5] Y. Fang, "A new general sufficient condition for almost sure stability of jump linear systems," IEEE Trans. Automat. Contr., vol. 42, pp. 378-382, Mar. 1997.

[6] D. Hristu-Varsakelis, "Feedback control systems as users of a shared network: communication sequences that guarantee stability," in Proc. 40th IEEE Conf. Decision and Control, Dec. 2001, pp. 3631-3636.

[7] H. Ishii and B. Francis, "Stabilizing a linear system by switching control and output feedback with dwell time," in Proc. 40th IEEE Conf. Decision and Control, Dec. 2001, pp. 191-196.

[8] F. Kozin, "A survey of stability of stochastic systems," Automatica, vol. 5, pp. 95-112, 1968.

[9] M. Mariton, Jump Linear Systems in Automatic Control. New York: Marcel Dekker, 1990.

[10] A. S. Matveev and A. V. Savkin, "Optimal control of networked systems via asynchronous communication channels with irregular delays," in Proc. 40th IEEE Conf. Decision and Control, Orlando, FL, Dec. 2001, pp. 2323-2332.

[11] L. A. Montestruque and P. J. Antsaklis, "Model-based networked control systems: Necessary and sufficient conditions for stability," presented at the 10th Mediterranean Conf. Control and Automation, Lisbon, Portugal, July 2002.

[12] — "State and output feedback control in model-based networked control systems," presented at the 41st IEEE Conf. Decision and Control, Las Vegas, NV, Dec. 2002.

[13] —_, "Stochastic stability for model-based networked control systems," presented at the 2003 American Control Conf., Denver, CO, June 2003.

[14] _ , "On the model-based control of networked systems," Automatica, vol. 39, pp. 1837-1843.

[15] M. Morari and E. Zafiriou, Robust Process Control, 1st ed. Upper Saddle River, NJ: Prentice-Hall, 1989.

[16] G. N. Nair, S. D. Dey, and R. J. Evans, "Communication-limited stabilisability of jump Markov linear systems," presented at the 15th Int. Symp. Mathematical Theory of Networks and Systems, Notre Dame, IN, Aug. 2002.

[17] Q. Ling and M. D. Lemmon, "Robust performance of soft real-time networked control systems with data dropouts," presented at the 41st IEEE Conf. Decision and Control, Las Vegas, NV, Dec. 2002.

[18] J. K. Yook, D. M. Tilbury, and N. R. Soparkar, "Trading computation for bandwidth: Reducing communication in distributed control systems using state estimators," IEEE Trans. Contr. Syst. Technol., vol. 10, pp. 503-518, July 2002.

[19] G. Walsh, H. Ye, and L. Bushnell, "Stability analysis of networked control systems," presented at the Amer. Control Conf., San Diego, CA, June 1999.

[20] S. Woods, Probability, Random Processes, and Estimation Theory for Engineers, 2nd ed. Upper Saddle River, NJ: Prentice-Hall, 1994. 


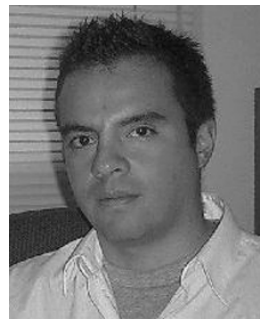

Luis A. Montestruque (M'02) received the B.S. degree in electrical engineering from the Pontifical Catholic University of Peru, Lima, in 1997, and the M.S. degree in electrical engineering from the University of Notre Dame, Notre Dame, IN, in 2001. He is currently working toward the Ph.D. degree in the Electrical Engineering Department, the University of Notre Dame.

Since 1995, he has worked in the design and implementation of industrial, environmental, and military-oriented networked embedded systems. His research interests include networked control and sensing systems and real-time embedded systems.

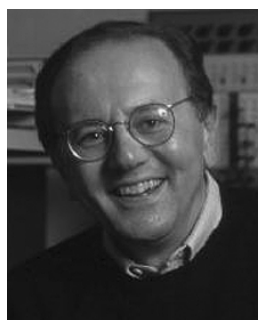

Panos Antsaklis (S'74-M'76-SM'86-F'91) received the undergraduate degree from the National Technical University of Athens (NTUA), Athens, Greece, and the M.S. and Ph.D. degrees from Brown University, Providence, RI.

$\mathrm{He}$ has held faculty positions at Brown University, Rice University, Houston, TX, and Imperial College of the University of London, London, U.K. During sabbatical leaves, he has lectured and conducted research at the Massachusetts Institute of Technology, Imperial College, NTUA, and the Technical University of Crete, Greece. He is the H.C. and E.A. Brosey Professor of Electrical Engineering and Director of the Center for Applied Mathematics at the University of Notre Dame, Notre Dame, IN. His work includes analysis of behavior and design of control strategies for complex autonomous and intelligent systems. His recent research focuses on networked embedded systems and addresses problems in the interdisciplinary research area of control, computing and communication networks, and on hybrid and discrete-event dynamical systems. He has authored a number of publications in journals, conference proceedings, and books, and he has edited several books on intelligent autonomous control and on hybrid systems. In addition, he has coauthored the research monograph Supervisory Control of Discrete Event Systems Using Petri Nets (Norwell, MA: Kluwer, 1998) and the graduate textbook Linear Systems (New York: McGraw-Hill, 1997).

Dr. Antsaklis serves on the editorial boards of several journals, and he currently serves as AEAL of the IEEE TRANSACTIONS AUTOMATIC CONTROL. He has been Guest Editor of Special Issues on Hybrid Systems in the IEEE Transactions Automatic CONTROL and the ProceEdings of the IEEE. He has served as Program Chair and General Chair of major systems and control conferences, as a Member and Chair of committees in the IEEE and IFAC, and he was the 1997 President of the IEEE Control Systems Society (CSS). He is a Distinguished Lecturer of the IEEE Control Systems Society, a recipient of the IEEE Distinguished Member Award of the Control Systems Society, and an IEEE Third Millennium Medal recipient. 